\title{
A fast wavelet algorithm for image deblurring
}

\author{
D. L. Donoho* M. E. Raimondo ${ }^{\dagger}$
}

(Received 8 October 2004, revised 1 February 2005)

\begin{abstract}
We present a nonlinear fully adaptive wavelet algorithm which can recover a blurred image $(n \times n)$ observed in white noise with $O\left(n^{2}(\log n)^{2}\right)$ steps. Our method exploits both the natural representation of the convolution operator in the Fourier domain and the typical characterisation of Besov classes in the wavelet domain. A particular feature of our method includes "cycle-spinning" band-limited wavelet approximations over all circulant shifts. The speed and the accuracy of the algorithm is illustrated with numerical examples of image deblurring. All figures presented in this paper are reproducible using the WaveD software package.
\end{abstract}

*Dept. Statistics, Stanford University, Stanford, U.S.A.

$\dagger^{\dagger}$ Dept. Maths \& Stats, The University of Sydney, Sydney, Australia. mailto:marcr@maths. usyd.edu . au

See http://anziamj.austms.org.au/V46/CTAC2004/Dono for this article, (c) Austral. Mathematical Soc. 2005. Published 10 March 2005, amended March 18, 2005. ISSN 1446-8735 


\section{Contents}

1 Introduction

C30

2 Preliminaries

C31

2.1 Two-dimensional wavelet bases . . . . . . . . . . C33

2.2 Translation Invariant wavelet transforms . . . . . . . . C C35

2.3 Periodised Meyer wavelets . . . . . . . . . . . . . C36

3 The two-dimensional WaveD-method $\quad$ C37

3.1 Fourier transforms . . . . . . . . . . . . . . . C37

3.2 The WaveD paradigm . . . . . . . . . . . . . C38

3.3 The WaveD estimator . . . . . . . . . . . C40

4 Examples

C41

4.1 WaveD Software. . . . . . . . . . . . . . C41

4.2 Image deblurring using WaveD . . . . . . . . . . . . C42

4.3 Results . . . . . . . . . . . . . . . . . C42

4.4 Discussion . . . . . . . . . . . . . . . . C43

5 Algorithm

C43

References

C46

\section{Introduction}

We begin with a function $f$ defined on the unit square $T=[0,1]^{2}$, this function represents our image. Let $g$ be a blurring kernel also defined on $T$ and let

$$
b(x):=f \star g(x)=\int_{T} f\left(x_{1}-u_{1}, x_{2}-u_{2}\right) g\left(u_{1}, u_{2}\right) d u_{1} d u_{2},
$$


represent our blurred image. Suppose we observe $b$ with some additive noise

$$
Y_{n}(d x)=b(x) d x+\sigma n^{-1} W(d x), \quad x \in T=[0,1] \times[0,1],
$$

where $\sigma$ is a positive constant and $W($.$) is a 2D Wiener process. Here and$ in the sequel of the paper $n^{-1}$ denotes the noise level for an image defined on a discrete set of equally spaced points $Y(i / n, j / n), i, j=1, \ldots, n$. This model is illustrated in Figure 1(b) using the well-known 'Camera' image, Figure 1(a). Our goal is to recover the original image $f$ from the noisy blurred observations (2) using a wavelet thresholding estimator. The model (2) is an idealised version of the motion blur model for digital images, see [1] for examples and applications.

In this paper we extend the WaveD paradigm of Johnstone, Kerkyacharian, Picard, and Raimondo [5] to the 2-dimensional setting and propose a non-linear fully adaptive wavelet estimator of the original image $f$. We show that the Translation-Invariant WaveD algorithm of Donoho and Raimondo [3] extends well to the 2-dimensional setting according to each orientation: vertical, horizontal and diagonal. This allows fast computation to be performed in the Fourier domain where the convolution operator (1) factorises nicely. Our paper is organised as follows: in Section 2 we review basic materials on 2-dimensional band-limited wavelet basis and translation invariant wavelet transforms; in Section 3, we present the 2-dimensional WaveD estimator; Section 4 is concerned with examples and numerical properties as well as a comparison with the recent ForWarD algorithm of [9]. A brief description of the 2-dimensional WaveD algorithm is given in Section 5.

\section{Preliminaries}


(a)

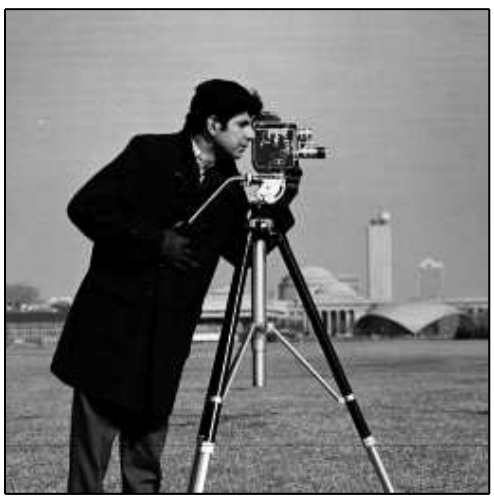

(c)

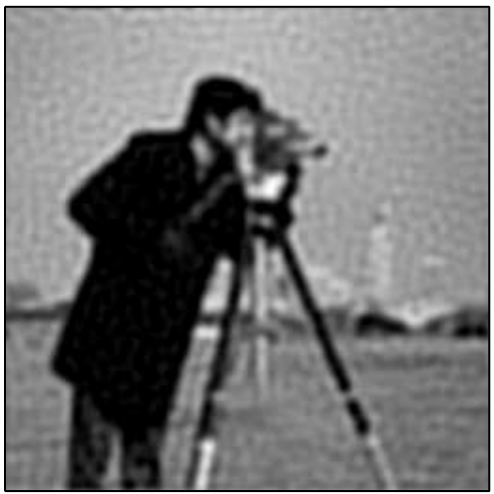

(b)

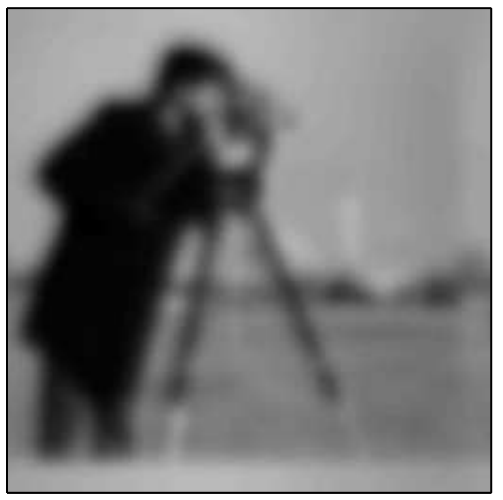

(d)

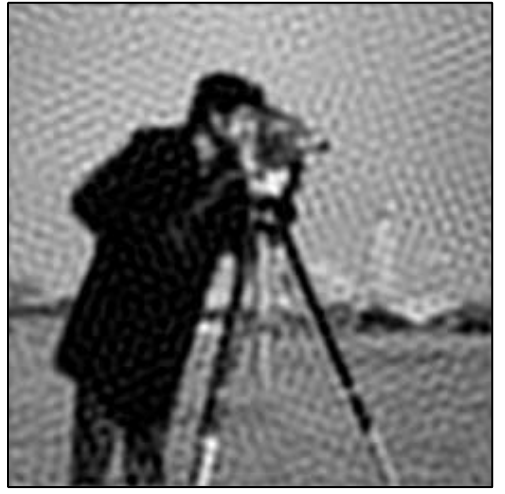

Figure 1: (a) Original image; (b) blurred and noisy image; (c) ForWard; (d) WaveD. 


\subsection{Two-dimensional wavelet bases}

Two-dimensional wavelet bases are constructed by tensor products of onedimensional wavelet orthogonal systems. We recall the following theorem taken from [7].

Theorem 1 Let $\phi$ be a scaling function and $\psi$ be the corresponding wavelet generating a wavelet orthonormal basis of $L^{2}(R)$. We define three wavelets:

$$
\psi^{1}(x)=\phi\left(x_{1}\right) \psi\left(x_{2}\right), \quad \psi^{2}(x)=\psi\left(x_{1}\right) \phi\left(x_{2}\right), \quad \psi^{3}(x)=\psi\left(x_{1}\right) \psi\left(x_{2}\right),
$$

and denote for $1 \leq m \leq 3, k=\left(k_{1}, k_{2}\right), x=\left(x_{1}, x_{2}\right)$ and $\kappa=(j, k)$

$$
\psi_{\kappa}^{m}(x):=\psi_{j, k}^{m}(x)=2^{j} \psi^{m}\left(2^{j} x_{1}-k_{1}, 2^{j} x_{2}-k_{2}\right) .
$$

The wavelet family

$$
\left\{\psi_{\kappa}^{1}, \psi_{\kappa}^{2}, \psi_{\kappa}^{3}\right\}_{\left(\kappa \in Z^{3}\right)}
$$

is an orthonormal basis of $L^{2}\left(R^{2}\right)$.

We illustrate this theorem using the Meyer wavelet [8]. A Meyer wavelet $\psi$ is a function whose Fourier transform $F(\psi):=\widehat{\psi}$ is smooth, see formula for the construction of $\hat{\psi}[7, \mathrm{p} .247]$. In practice, we use a polynomial function to define the so-called Meyer window [7, p.248]. Note that the Meyer wavelet is band limited and we have $\operatorname{Supp}(\widehat{\phi}(w))=\{w:|w| \in[0,4 \pi / 3]\}$ and $\operatorname{Supp}(\widehat{\psi}(w))=\{w:|w| \in[2 \pi / 3,8 \pi / 3]\}$.

Applying Fourier transforms in (3) we construct two-dimensional Meyer wavelets in the Fourier domain $\omega=\left(w_{1}, w_{2}\right)$,

$$
\widehat{\psi}^{1}(\omega)=\widehat{\phi}\left(w_{1}\right) \widehat{\psi}\left(w_{2}\right), \quad \widehat{\psi}^{2}(\omega)=\widehat{\psi}\left(w_{1}\right) \widehat{\phi}\left(w_{2}\right), \quad \widehat{\psi}^{3}(\omega)=\widehat{\psi}\left(w_{1}\right) \widehat{\psi}\left(w_{2}\right)
$$

and $\widehat{\phi}(\omega)=\widehat{\phi}\left(w_{1}\right) \widehat{\phi}\left(w_{2}\right)$ for the two-dimensional scaling function. This process is illustrated in Figure 2. 
(a)

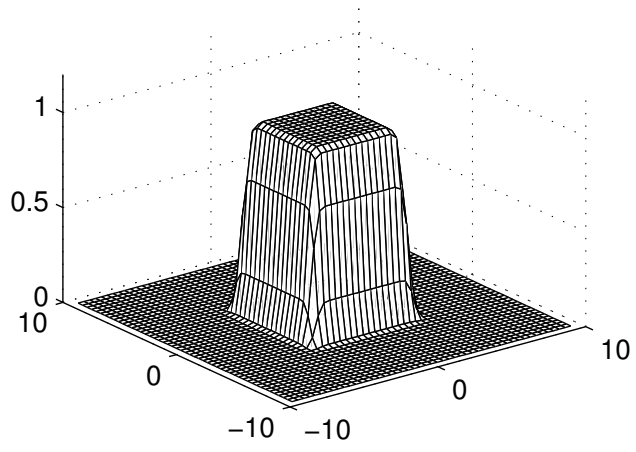

(c)

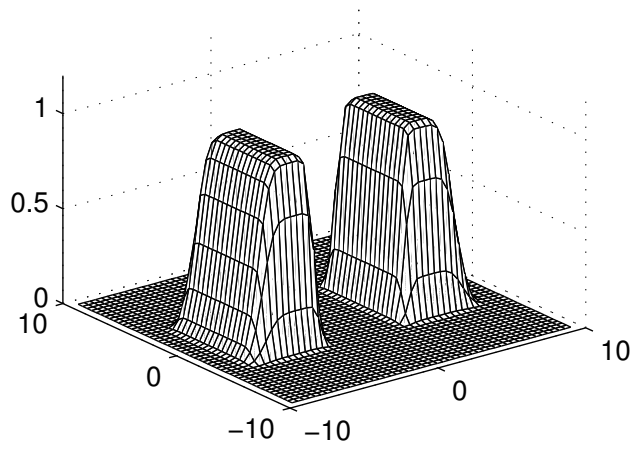

(b)

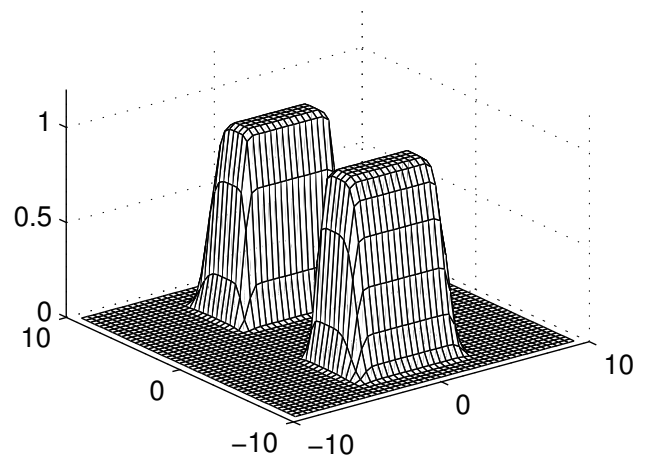

(d)

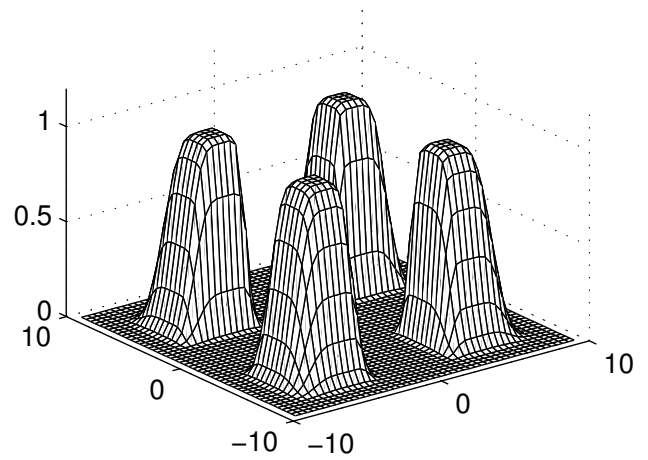

Figure 2: (a) $|\widehat{\phi}(\omega)|$; (b) $\left|\widehat{\psi}^{1}(\omega)\right|$; (c) $\left|\widehat{\psi}^{2}(\omega)\right|$; (d) $\left|\widehat{\psi}^{3}(\omega)\right|$. 
The three wavelets defined in Theorem 1 will reveal image details at different frequencies and orientations: $\psi_{\kappa}^{1}$ will extract vertical details; $\psi_{\kappa}^{2}$ will extract horizontal details; and $\psi_{\kappa}^{3}$ will extract diagonal details. This orientation property is visible in the Fourier domain, for example $\left|\psi_{\kappa}^{1}(\omega)\right|$ will be large at low horizontal frequencies and high vertical frequencies, see Figure $2(\mathrm{~b})$.

\subsection{Translation Invariant wavelet transforms}

In this section we recall the formal definition of cycle-spinning a generic (twodimensional) wavelet thresholding estimator and we define the Translation Invariant (TI) WaveD estimator. We refer to [2] and references therein for a more general discussion. A wavelet thresholding estimator is represented as

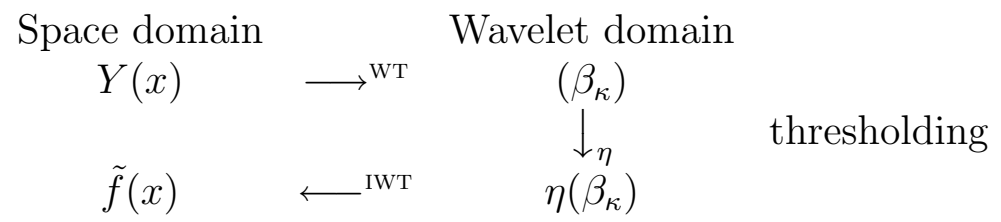

where WT, IWT denotes a generic wavelet transform and its inverse. For any space shift $h=\left(h_{1}, h_{2}\right)$, we let $T_{h} f(x)=f(x+h):=f\left(x_{1}+h_{1}, x_{2}+h_{2}\right)$ denote the shift operator. For an arbitrary shift $h$ we define one cycle-spin of the estimator $\tilde{f}(t)$ as

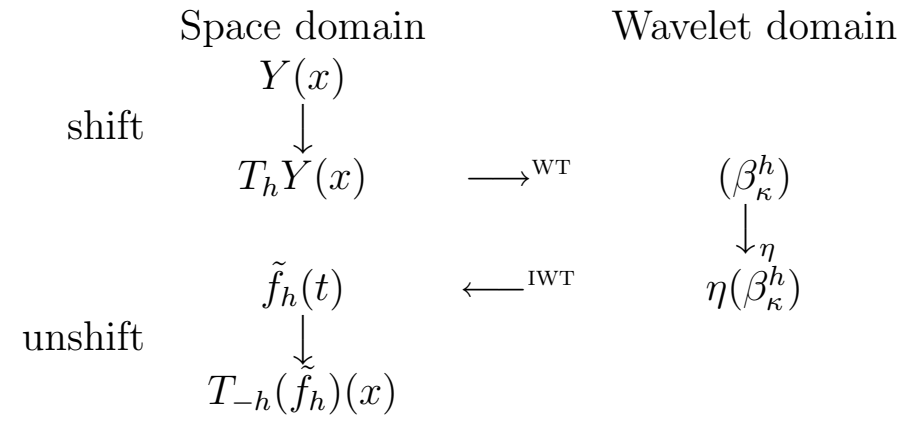

thresholding 
The idea behind cycle-spinning is to correct possible mis-alignments between features in the image and features in the basis. Of course, if the image is unknown there is little hope to find the best value of $h$. A well established approach to solve this problem is to apply a range of shifts and average over all results [2]. Let $H$ be a set of shifts, cycle-spinning the WaveD estimator over $H$ yields a new estimator:

$$
\tilde{f}_{H}=\operatorname{Ave}_{h \in H} T_{-h}\left(\tilde{f}_{h}\right)=\frac{1}{|H|} \sum_{h \in H} T_{-h}\left(\tilde{f}_{h}\right),
$$

where $|H|$ denotes the cardinality of $H$. Yet again the problem of choosing the best $H$ may arise. In fact, it is clear that the bigger $H$ is the better since we take into account more possible mis-alignments. Assuming that the observed data $y=\left(y_{(1,1)}, \ldots, y_{(i, j)}, \ldots, y_{(n, n)}\right)$ are sampled at a discrete set of equally spaced points $y_{(i, j)}=Y(i / n, j / n), i, j=1, \ldots, n$; we define $H_{1}=\{1 / n, 2 / n, \ldots, n-1 / n, 1\}$ and $H_{2}=H_{1} \times H_{1}$ to be set of all possible circulant shifts. The Translation Invariant WaveD estimator is defined by

$$
\tilde{f}_{\mathrm{TI}}=\operatorname{Ave}_{h \in H_{2}} T_{-h}\left(\tilde{f}_{h}\right) .
$$

We refer to Section 4 of [2] for a discussion of the improvement provided by the cycle spinning process. This includes for example improvement in approximation, suppression of Gibbs phenomenon as well as improvement in de-noising.

\subsection{Periodised Meyer wavelets}

We start with a 1-dimensional Meyer orthogonal system $\phi, \psi$ and let

$$
\Psi(x)=\sum_{k \in Z} \psi(x+k), \quad \Psi_{j, k}(x)=2^{j / 2} \Psi\left(2^{j} x-k\right),
$$

with a similar formula for the periodised scaling function $\Phi$ in terms of the Meyer scaling function $\phi$. It is easy to check that $(\Phi, \Psi)$ are periodic and yield 
an orthonormal basis of $L^{2}(T)$, see [4]. The next step is to apply Theorem 1 to the periodised pair $(\Phi, \Psi)$ which gives a two-dimensional periodised wavelet basis of $L^{2}(T)$ :

$$
\left\{\Psi_{\kappa}^{0}, \Psi_{\kappa}^{1}, \Psi_{\kappa}^{2}, \Psi_{\kappa}^{3}\right\}_{\left(\kappa \in Z^{3}\right)}
$$

where $\Psi_{\kappa}^{0}:=\Phi_{\kappa}$ denotes the scaling function.

\section{The two-dimensional WaveD-method}

\subsection{Fourier transforms}

For $l=\left(l_{1}, l_{2}\right) \in Z^{2}$ and $x=\left(x_{1}, x_{2}\right) \in T$, let $l^{\prime} x=l_{1} \cdot x_{1}+l_{2} \cdot x_{2}$ and $e_{l}(x)=\exp \left(2 \pi i l^{\prime} x\right), l \in Z^{2}$ denote the Fourier basis of $L^{2}(T)$. For periodic functions (images) of $L^{2}(T)$, the Fourier coefficients

$$
f_{l}:=\left\langle f, e_{l}\right\rangle=\int_{0}^{1} \int_{0}^{1} f\left(x_{1}, x_{2}\right) e_{l}\left(x_{1}, x_{2}\right) d x_{1} d x_{2} .
$$

For blurred images $b=f \star g$, we have the nice formula

$$
b_{l}:=\left\langle b, e_{l}\right\rangle=\left\langle f \star g, e_{l}\right\rangle=\left\langle f, e_{l}\right\rangle \cdot\left\langle g, e_{l}\right\rangle=f_{l} . g_{l},
$$

for noisy images $Y_{n}(x)$ and Wiener process $W(x)$ the Fourier coefficients are

$$
\begin{aligned}
& y_{l}=\left\langle Y_{n}, e_{l}\right\rangle:=\int_{0}^{1} \int_{0}^{1} e_{l}\left(x_{1}, x_{2}\right) d Y_{n}\left(x_{1}, x_{2}\right), \\
& z_{l}=\left\langle W, e_{l}\right\rangle:=\int_{0}^{1} \int_{0}^{1} e_{l}\left(x_{1}, x_{2}\right) d W\left(x_{1}, x_{2}\right) .
\end{aligned}
$$

Applying the two-dimensional Fourier transform to the data (2) gives

$$
y_{l}=b_{l}+\sigma n^{-1} z_{l}, \quad l \in Z^{2} .
$$


The WaveD paradigm [5] combines Fourier and wavelet transforms. For periodised wavelets (10), the Fourier coefficients

$$
\Psi_{l}^{m, \kappa}:=\left\langle\Psi_{\kappa}^{m}, e_{l}\right\rangle=\int_{0}^{1} \int_{0}^{1} \Psi_{\kappa}^{m}\left(x_{1}, x_{2}\right) e_{l}\left(x_{1}, x_{2}\right) d x_{1} d x_{2},
$$

it is straightforward to check that the latter Fourier coefficients can be computed from the Fourier transform of the original wavelet (evaluated on a dyadic grid). For example,

$$
\Psi_{l}^{m, j, 0,0}=\left\langle\Psi_{j, 0,0}^{m}, e_{l}\right\rangle=2^{-j} \widehat{\psi}^{m}\left(l /\left(2^{j} .2 \pi\right)\right),
$$

using the construction formula for $\widehat{\psi}[7$, p.247], we compute the Fourier coefficients of $\Psi_{j, 0,0}^{m}$ at any resolution level $j$. This is illustrated on Figure 3 with $j=3$ and $m=2$.

Remark 1. The Fourier coefficients (17) whose modulus are depicted on Figure 3(b) play a key role in the WaveD algorithm. Proposition 2 (Section 5) shows that the knowledge of these coefficients allows a fast computation of the complete wavelet transform (and its inverse) at resolution level $j$, see Figure 4.

\subsection{The WaveD paradigm}

For periodic images in $L^{2}(T)$, the wavelet coefficients

$$
\beta_{\kappa}^{m}:=\left\langle f, \Psi_{\kappa}^{m}\right\rangle=\int_{0}^{1} \int_{0}^{1} f\left(x_{1}, x_{2}\right) \Psi_{\kappa}^{m}\left(x_{1}, x_{2}\right) d x_{1} d x_{2} .
$$

For blurred images $b=f \star g$, we compute the wavelet transform via Plancherel's formula:

$$
\left\langle b, \Psi_{\kappa}^{m}\right\rangle=\sum_{l} b_{l} \cdot \bar{\Psi}_{l}^{m, \kappa},
$$


(a)

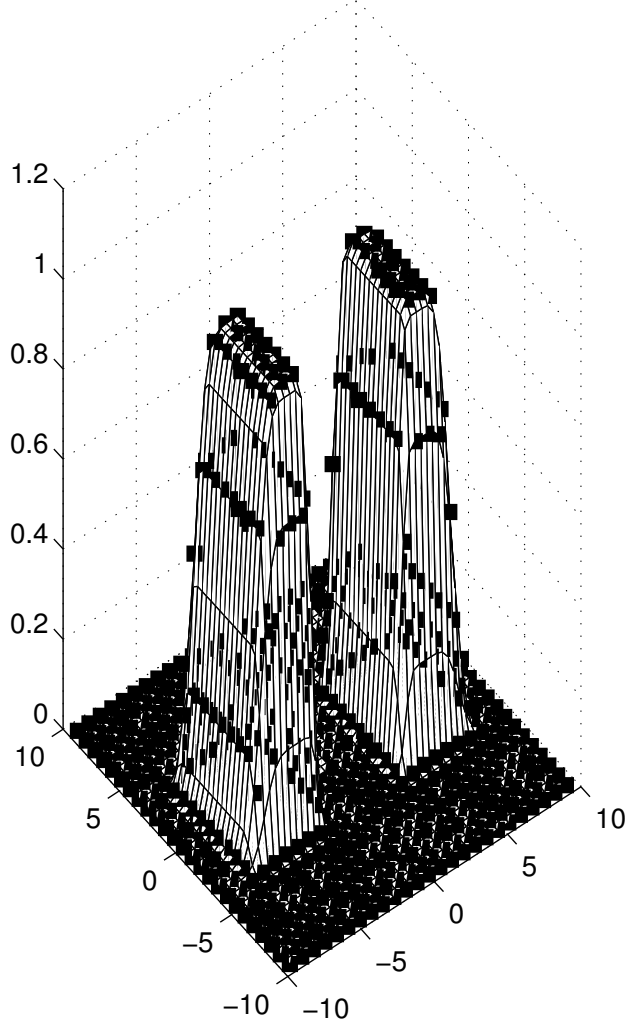

(b)

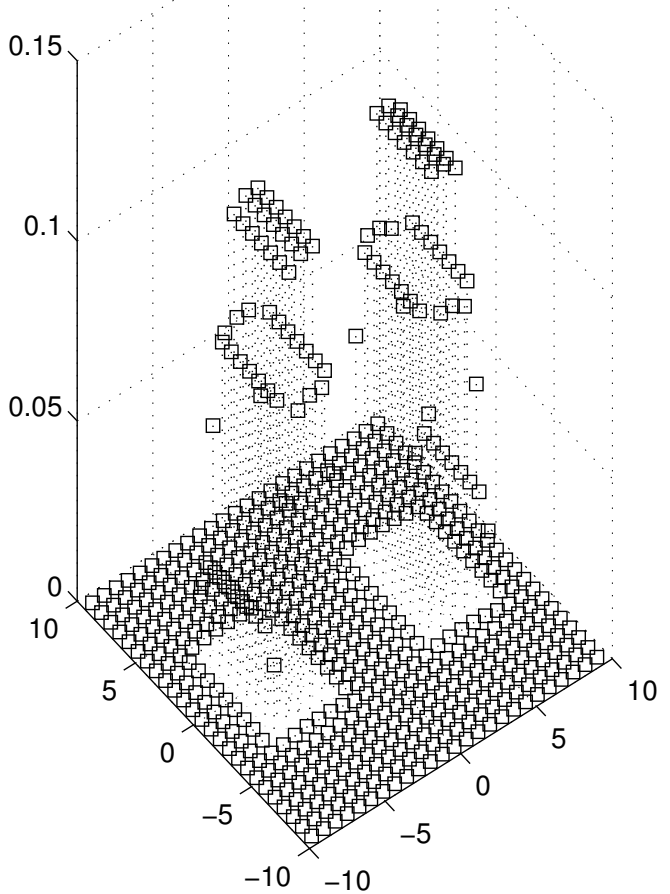

Figure 3: (a) The continuous surface depicts $\omega \rightarrow\left|\hat{\psi}^{2}(\omega)\right|$ and points are sampling at $\omega_{l}=l /\left(2^{j} .2 \pi\right), l \in Z$, with resolution level $j=3$; (b) Modulus of Fourier coefficients $\Psi_{l}^{m, j, 0,0}=\hat{\psi}^{2}\left(\omega_{l}\right) / 8$, with $m=2$ and $j=3$, see (17). 
the WaveD paradigm stipulates that we can perform wavelet transform and deconvolution simultaneously, combining (12) and (19) with Plancherel's equality:

$$
\operatorname{WaveD}\left(b, g, \Psi_{\kappa}^{m}\right):=\sum_{l}\left(\frac{b_{l}}{g_{l}}\right) \cdot \bar{\Psi}_{l}^{m, \kappa}=\sum_{l} f_{l} \cdot \bar{\Psi}_{l}^{m, \kappa}=\beta_{\kappa}^{m} .
$$

In the case of noisy images, we define the (unbiased) Waved estimator

$$
\tilde{\beta}_{\kappa}^{m}=\operatorname{WaveD}\left(Y, g, \Psi_{\kappa}^{m}\right):=\sum_{l \in C_{j}^{m}}\left(\frac{y_{l}}{g_{l}}\right) \bar{\Psi}_{l}^{m, \kappa},
$$

where $C_{j}^{m}:=\left\{l: \Psi_{l}^{m, \kappa} \neq 0\right\}$. In the light of the above WaveD formula, we see that band-limited wavelets (for example, Meyer) are very attractive since only a finite number of terms is used in the sum (21).

\subsection{The WaveD estimator}

After performing deconvolution and wavelet transform, according to (21), we define the WaveD estimator

$$
\tilde{f}=\sum_{\kappa \in I_{0}} \tilde{\beta}_{\kappa}^{0} \mathbf{I}_{\left\{\left|\tilde{\beta}_{\kappa}\right| \geq \lambda_{j_{0}}\right\}} \Psi_{\kappa}^{0}+\sum_{m=1}^{3} \sum_{\kappa \in I_{1}} \tilde{\beta}_{\kappa}^{m} \mathbf{I}_{\left\{\left|\tilde{\beta}_{\kappa}^{m}\right| \geq \lambda_{j}^{m}\right\}} \Psi_{\kappa}^{m},
$$

where $\mathbf{I}$ denotes the indicator function and $I_{0}, I_{1}$ are set of indices; $I_{0}=$ $\left\{\left(j_{0}, k_{1}, k_{2}\right): 0 \leq k_{i} \leq 2^{j_{0}}-1, i=1,2\right\}$ corresponds to a coarse resolution level $j_{0}$ and $I_{1}=\left\{\left(j, k_{1}, k_{2}\right): 0 \leq k_{i} \leq 2^{j}-1, i=1,2, j_{0} \leq j \leq j_{1}\right\}$ indexes details up to a fine resolution level $j_{1}$. To specify the thresholds $\lambda_{j}^{m}$, we extend the definition of [5] to the case of an image defined on a discrete set of equally spaced points $f(i / n, j / n), i, j=1, \ldots, n$ and let

$$
\tau_{j}^{m}=\left(\left|C_{j}^{m}\right|^{-1} \sum_{l \in C_{j}^{m}}\left|g_{l}\right|^{-2}\right)^{1 / 2},
$$


where $\left|C_{j}^{m}\right|$ denotes the cardinality of $C_{j}^{m}$. The WaveD (level-by-level) thresholds are defined according to each orientation $(m=0,1,2,3)$ by

$$
\lambda_{j}^{m}:=\gamma \sigma \tau_{j}^{m}(\log n) / n,
$$

where $\gamma$ is a constant and the finest scale $j_{1}$ is determined from

$$
2^{j_{1}}=(n / \log n)^{1 /(1+2 \nu)} .
$$

Here $\nu$ is a decay parameter which depends on the Degree of Ill-Posedness (DIP) of the convolution model (2); for example the WaveD asymptotic theory as prescribed in [5] (extended here to the 2D-setting) includes convolution kernel $g$ such that

$$
\left(\left|C_{j}^{m}\right|^{-1} \sum_{l \in C_{j}^{m}}\left|g_{l}\right|^{-2}\right)^{1 / 2} \asymp 2^{j \nu} .
$$

The notation $a_{j} \asymp b_{j}$ means that there exist constants $c_{0}, c_{1}$ such that for all $j, c_{0} \leq a_{j} / b_{j} \leq c_{1}$. Condition $\left(C_{\nu}\right)$ typically holds for ordinary "smooth" convolution where $\left|g_{l}\right| \sim\left(\left|l_{1}\right|+\left|l_{2}\right|\right)^{-\nu}$, and in the 1-dimensional setting it has been shown to hold for certain oscillatory cases, such as arise with irrational boxcar blur [6].

\section{Examples}

\subsection{WaveD Software}

The WaveD2.0 software package used to prepare all figures and table in this paper is available at http://www. maths. usyd.edu.au:8000/u/marcr/. The 2D-WaveD algorithm as described in Section 5 is implemented in MatlaB and is based on the repeated application of appropriate discrete Fourier transform at each resolution level (and orientation) as illustrated in Figure 4. For an $n \times n$ image there are at most $J=\log _{2}(n)-1$ resolution levels and each 2D-Fourier transform takes $O\left(n^{2} \log n\right)$ steps. Hence, in total the 2D-WaveD algorithm requires only $O\left(n^{2}(\log n)^{2}\right)$ steps for an image with $n \times n$ pixels. 
TABlE 1: Monte-Carlo approximations to RMISE $=\sqrt{E\|\tilde{f}-f\|_{2}^{2}}$ with $N=$ 100 replications.

\begin{tabular}{|cc|ccc|}
\hline Method & Time $(100$ repl. $)$ & Low noise & Medium noise & High noise \\
\hline WaveD & $4.5 \mathrm{~min}$ & 0.1854 & 0.1886 & 0.2442 \\
ForWaRD & $55.8 \mathrm{~min}$ & 0.1581 & 0.1812 & 0.2953 \\
\hline
\end{tabular}

\subsection{Image deblurring using WaveD}

We have tested finite sample properties of the WaveD algorithm using a wide range of images at various Signal-to-Noise-Ratios. We illustrate our findings using the well-known 'Camera' image, see [9, e.g.]. The original image $(256 \times 256)$ is depicted in Figure 1(a). In Figure 1(b) we illustrate the white noise model (2) with medium noise level $\sigma_{\text {med }}=0.002$ and a smooth blurring kernel $g$ with DIP $\nu=1$. In Figure (b) the Blurred-Signal-toNoise-Ratio (BSNR) is $40 \mathrm{~dB}$ as in the default setting of [9] . In our study (summarised in Table 1) we also report performances for low noise level $\sigma_{\text {low }}=0.0006(\mathrm{BSNR}=50 \mathrm{~dB})$ and high noise level $\sigma_{\text {high }}=0.065(\mathrm{BSNR}=$ $10 \mathrm{~dB})$.

\subsection{Results}

For each combination of noise level (low, medium and high) we compute the Monte Carlo approximation to the Root-Mean-Integrated-Square-Error (RMISE) with $N=100$ replications. For comparison, we include the results of the recently developed ForWard algorithm, see [9].

The general pattern seen in Table 1 suggests that for high noise level WaveD outperforms ForWarD whereas for low noise level ForWarD outperforms WaveD. For medium noise level both WaveD and ForWaRD are very 
close. A comparison of Figure 1(c) and 1(d) suggests that the image details (for example, eyes, mouth) are more visible on the WaveD estimation than in the ForWarD image which remains a little blurred due to the regularisation. On the other hand the general appearance of the ForWarD estimates is slightly smoother than the WaveD image.

\subsection{Discussion}

These preliminary results are very encouraging given that the ForWarD method is known to have very good numerical properties and outperforms classical deblurring methods based on Wiener filtering. A key feature of the WaveD method is that it is fairly simple and it enjoys fast computation (nearly as fast as the fast Fourier Transform). Our numerical study seems to suggest that diagonal coefficients at large resolution level are harder to recover than horizontal or vertical components. This is confirmed by the theory since an inspection of the WaveD paradigm (21) shows that for diagonal coefficients $(m=3)$ the support of $\hat{\Psi}^{3}$ is wider than that of $\hat{\Psi}^{m}$, $m=1,2$ (see also Figure 3). This yields larger variances for diagonal coefficients estimates. This results in a lack of diagonal coefficients for the WaveD image (Figure 1(d)). There is a similar phenomenon in ForWarD images (Figure 1(c)) but the regularisation tends to minimise this effect although this also erases some details in the image. We are confident that the general aspect of WaveD images could further be improved by using more elaborated thresholding strategies, see for example the discussion in [5].

\section{Algorithm}

Our algorithm is based on a mathematical idea introduced in [3] for the 1dimensional setting. The idea is that, at a given resolution level, both wavelet transform and inverse wavelet transform can be expressed as convolutions 
with certain wavelet functions. The WaveD algorithm takes full advantage of the Fast Fourier Transform to compute those convolutions. Below we present an extension of the WaveD algorithm to the 2-dimensional setting where we take care of the 3 possible orientations: horizontal, vertical and diagonal.

- Averaging shifts can be done level by level according to given orientation $m=1,2$ and 3 . To simplify the presentation we omit the scaling term $m=0$. Recalling the multi-resolution structure of a generic wavelet estimator we have

$$
\begin{gathered}
\left(T_{-h} f_{h}^{m}(x)\right)=\sum_{j=j_{0}}^{j_{1}} \sum_{k \in I_{j}^{m}} \eta\left(\tilde{\beta}_{j, k}^{m, h}\right) T_{-h} \Psi_{j, k}^{m}(x):=\sum_{j=j_{0}}^{j_{1}} P_{j}^{m, h}(x) . \\
f_{H}^{m}(x)=\operatorname{Ave}_{h \in H}\left(T_{-h} f_{h}^{m}(x)\right)=\sum_{j=j_{0}}^{j_{1}} A v e_{h \in H} P_{j}^{m, h}(x):=\sum_{j=j_{0}}^{j_{1}} f_{j}^{m, H}(x),
\end{gathered}
$$

finally we sum the horizontal, vertical and diagonal contributions:

$$
f_{H}(x)=\sum_{m=1}^{3} f_{H}^{m}(x) .
$$

- For the TI-estimator the approximations $f_{j}^{m, H}(m=1,2,3)$ can be expressed in terms of convolutions with certain wavelet functions. The following result is a direct extension of Proposition 1 of [3].

Proposition 2 Let $k=\left(k_{1}, k_{2}\right)$ and $\tilde{\Psi}_{j, k}^{m}(x)=\Psi_{j, k}^{m}(-x)$. For any resolution level $j$ and shift $h=\left(h_{1}, h_{2}\right)$ we have, for $m=1,2$ and 3 ,

$$
\beta_{j, k}^{m, h}=\left(f \star \tilde{\Psi}_{j, 0,0}^{m}\right)\left(\Delta_{k}^{h}\right):=A_{j}^{m}\left(\Delta_{k}^{h}\right),
$$




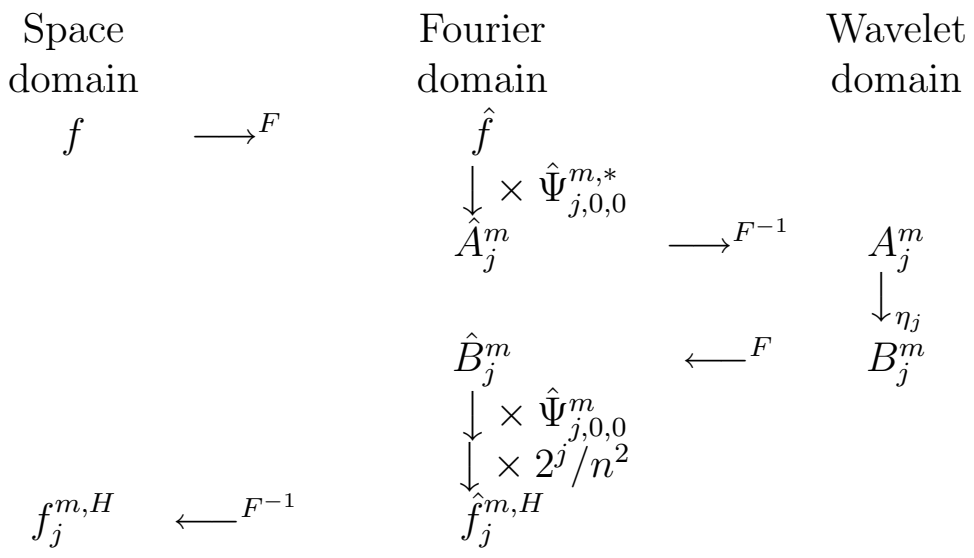

\section{Figure 4:}

where $\Delta_{k}^{h}=k / 2^{j}+h$. Let $H$ be the range of shifts defined by $H=$ $\{1 / n, 2 / n, \ldots,(n-1) / n\} \times\{1 / n, 2 / n, \ldots,(n-1) / n\}$, then,

$$
f_{j}^{m, H}(x)=\left(2^{j} / n^{2}\right) \times\left(B_{j} * \Psi_{j, 0,0}^{m}\right)(x),
$$

where

$$
B_{j}^{m}\left(\Delta_{k}^{h}\right):=\eta_{j}\left(A_{j}^{m}\left(\Delta_{k}^{h}\right)\right)=A_{j}^{m}\left(\Delta_{k}^{h}\right) \cdot \mathbf{I}\left(\left|A_{j}^{m}\left(\Delta_{k}^{h}\right)\right|>\lambda_{j}^{m}\right),
$$

and $f * g$ denotes the circular convolution product.

The convolutions (27) and (28) are computed in the Fourier domain while the thresholding process (29) is done in the wavelet domain. This is illustrated in Figure 4 where $F, F^{-1}$ denotes the Fourier transform and its inverse; $\hat{\Psi}_{j, 0,0}^{m}$ denotes the Fourier coefficients of $\Psi_{j, 0,0}^{m}$ and $\hat{\Psi}_{j, 0,0}^{m, *}$ denotes their complex conjugates (Hermitian symmetry is used in (27)).

Acknowledgment: We are grateful to two referees for helpful comments. 


\section{References}

[1] M. Bertero and P. Boccacci, Introduction to Inverse Problems in Imaging, Institute of Physics, Bristol and Philadelphia, 1998. C31

[2] R. Coifman and D. Donoho, Translation-invariant de-noising, in Wavelets and Statistics, A. Antoniadis and G. Oppenheim, eds., New York: Springer Verlag, vol. 103 of Lecture Notes in Statistics (1995) pp. 125-150, pp. 125-150. C35, C36

[3] D. Donoho and M. Raimondo, Translation invariant deconvolution in a periodic setting, The International Journal of Wavelets, Multiresolution and Information Processing 2, No. 4 (2004), 415-431. C31, C43, C44

[4] E. Hernàndez and G. Weiss, A First Course on Wavelets, CRC Press, 1996. C37

[5] I. Johnstone, G. Kerkyacharian, D. Picard, and M. Raimondo, Wavelet deconvolution in a periodic setting, Journal of the Royal Statistical Society, Series B 66 (2004), 547-573. With discussion pp.627-652. C31, C38, C40, C41, C43

[6] I. M. Johnstone and M. Raimondo, Periodic boxcar deconvolution and diophantine approximation, Annals of Statistics 32 (2004), 1781-1804. C41

[7] S. Mallat, A wavelet tour of signal processing (2nd Edition), Academic Press Inc., San Diego, CA, 1998. C33, C38

[8] Y. Meyer, Ondelettes et Opérateurs-I, Hermann, 1990. C33

[9] R. Neelamani, H. Choi, and R. Baraniuk, Forward: Fourier-wavelet regularized deconvolution for ill-conditioned systems, IEEE Transactions on signal processing 52 (2004), 418-433. C31, C42 